\title{
LHOMME L'Homme
}

Revue française d'anthropologie

$200 \mid 2011$

Décrire, écrire

\section{Ethno-graphies}

Éric Jolly et Marianne Lemaire

\section{OpenEdition}

Journals

Édition électronique

URL : http://journals.openedition.org/lhomme/22831

DOI : $10.4000 /$ /homme.22831

ISSN : 1953-8103

\section{Éditeur}

Éditions de l'EHESS

\section{Édition imprimée}

Date de publication : 21 novembre 2011

Pagination : 13-17

ISSN : 0439-4216

\section{Référence électronique}

Éric Jolly et Marianne Lemaire, «Ethno-graphies », L'Homme [En ligne], 200 | 2011, mis en ligne le 09 novembre 2013, consulté le 01 mai 2019. URL : http://journals.openedition.org//homme/22831 ; DOI : 10.4000//homme.22831

Ce document a été généré automatiquement le 1 mai 2019.

(c) École des hautes études en sciences sociales 


\title{
Ethno-graphies
}

\author{
Éric Jolly et Marianne Lemaire
}

1 INFLUENCÉE PAR le contexte disciplinaire, l'écriture d'un ethnologue garde néanmoins la trace du parcours personnel de son scripteur. Lorsqu'ils sont mis en regard, textes, biographies et histoire scientifique s'éclairent ainsi mutuellement. Les quatre articles de ce dossier le démontrent en examinant les écrits de plusieurs ethnographes (ou groupes d'ethnographes) à partir d'une double histoire: celle de leur discipline et celle, plus personnelle, de leurs habitudes, goûts, stratégies de lecture et d'écriture. Relevant d'échelles différentes, ces histoires ne sont en rien indépendantes. Par le biais de leurs travaux, Paul Rivet, Marcel Griaule, Denise Paulme ou l'équipe de Minot ont contribué à l'évolution des modèles de notation et de description ethnographiques, mais, inversement, les nouvelles normes de l'ethnologie française ont pesé sur les choix rédactionnels de ces chercheurs et, à la suite de leurs choix, sur leur carrière. Ces ethnographes ne sont donc ni des serviteurs obséquieux d'une écriture conventionnelle ni de simples rhéteurs ou des écrivains affranchis de toutes contraintes. Il convient plutôt de les considérer comme des acteurs singuliers susceptibles d'adapter (voire d'abandonner) leurs habitudes et leurs préférences graphiques, en fonction du contexte, avec en définitive des résultats variés et contrastés : polygraphie ou registre discursif unique, écriture dépouillée ou flamboyante, texte synthétique ou narratif, énoncé collectif ou individuel...

\section{Écritures et stratégies de légitimation}

2 D'après les études rassemblées ici, plusieurs éléments biographiques sont susceptibles d'expliquer les choix scripturaux d'un ethnologue aux différentes étapes de sa carrière : son identité sexuelle, son origine sociale, sa formation, ses influences littéraires, son expérience du terrain et, enfin - peut-être surtout -, sa position institutionnelle. En effet, pour les ethnologues français de la première moitié $\mathrm{du} \mathrm{xx}^{\mathrm{e}}$ siècle, l'écriture devient un élément important de leur stratégie de légitimation et d'insertion professionnelles ${ }^{1}, a$ fortiori lorsqu'ils débutent. Plutôt normatifs, de tels choix se manifestent alors en creux à 
travers les renoncements et les interdits que les chercheurs s'imposent pour faire la preuve de leur rigueur et de leurs compétences. Au tout début du $\mathrm{xx}^{\mathrm{e}}$ siècle, un jeune anthropologue devait ainsi choisir entre science et poésie pour des raisons de crédibilité et de légitimité, comme le montre Christine Laurière grâce à une analyse fine et sensible de plusieurs poèmes inédits de Paul Rivet, tous composés lors de sa première mission en Équateur. Jeune médecin militaire en cours de reconversion, Rivet se refuse à publier ces écrits intimes qui, dans leur forme comme dans leur contenu, risquent de le disqualifier aux yeux des anthropologues alors qu'il tente de forcer leur reconnaissance en s'appropriant leurs codes.

3 Marianne Lemaire montre également que, pour s'affirmer comme scientifique et prétendre aux mêmes postes que les hommes, les femmes ethnographes des années 1930 s'autorisent uniquement une écriture dépourvue d'affects, d'effets littéraires et de mise en scène de soi, contrairement à leurs collègues masculins qui, loin de s'effacer, multiplient récits de voyage ou journaux intimes². Rédhibitoire pour les femmes, l'association entre ethnologie et aventure reste en effet acceptable pour les hommes, du moins à cette époque.

4 Analysé par Éric Jolly, l'exemple de Marcel Griaule offre d'ailleurs un exemple du versant masculin de cette écriture ethnographique des années 1930. Si les grandes monographies de Griaule sont conformes aux standards de la discipline, la légitimité et la renommée scientifiques de ce chef de mission se nourrissent avant tout d'une autorité acquise sur le terrain, puis mise en scène par écrit. Au lieu d'exclure récits de voyage et chroniques d'enquêtes, Griaule en use ainsi généreusement pour promouvoir sa discipline et sa carrière, en combinant différents types de textes ou d'images et en les adaptant à ses goûts graphiques.

5 Dans le cas de l'étude réalisée à Minot entre 1968 et 1975, les usages de l'écrit répondent là encore à des exigences de légitimation, mais en fonction d'enjeux disciplinaires nouveaux. Revenant sur cette fameuse enquête collective dont elle était l'une des quatre participantes, Françoise Zonabend témoigne avec acuité et retenue des choix solidaires effectués par les membres de cette mission, et ce à toutes les étapes de leur travail: partage de leurs notes, publications à plusieurs mains et harmonisation de leurs écritures respectives. De telles pratiques visaient notamment à recouper leurs sources et à « parler d'une même voix ", chacune sur des sujets différents, afin de renforcer la cohérence et la crédibilité de ces recherches, en démontrant ainsi aux américanistes, aux africanistes, aux océanistes qu'une ethnologie du proche était possible ${ }^{3}$. Selon le contexte et le chercheur, la quête de légitimité passe donc par des stratégies différentes et par une grande variété de registres rédactionnels.

\section{Choix scripturaux et résultats scientifiques}

6 Autre enseignement de ce dossier : les partis pris graphiques d'un ethnographe sont aussi des choix intellectuels liés à des orientations méthodologiques, thématiques et théoriques. Alors que Rivet, à travers ses poèmes, évoque avec émotion la misère et l'exploitation des Amérindiens, son renoncement à toute licence poétique traduit son recentrement vers un objet d'étude plus légitime, en partie construit par l'ethnographie de l'époque : l'Indien « pur » et lointain, préservé de la contamination de l'Occident ou du monde urbain. Un tel paradigme, note Christine Laurière, explique la triple autocensure - 
stylistique, affective et thématique - à laquelle Rivet se soumet. Figure centrale de l'article de Marianne Lemaire, Denise Paulme se fixe des limites différentes : bannissant toute fantaisie littéraire, elle privilégie une description précise de la vie économique et sociale en évitant à la fois les objets d'étude spectaculaires et ceux réservés intuitivement aux femmes. La rigueur de sa présentation se conjugue ainsi avec la «neutralité» volontaire de ses thématiques de recherche afin d'échapper aux stéréotypes sur la femme ethnographe. Dans son article sur Minot, Françoise Zonabend s'interroge également sur les blancs ou les trous de l'enquête en les expliquant par des engagements collectifs articulés eux-mêmes à des histoires personnelles. L'effacement volontaire de chaque chercheuse au profit du groupe s'est traduit par des écrits qui se raccordent et "s'ajustent les uns aux autres ", mais ce sont des écrits respectueux des intérêts, des affects et des parcours de chacune. Parce qu'il risquait de rouvrir des blessures impossibles à partager, un sujet comme l'Occupation a été ainsi éludé pour préserver la cohésion de l'équipe.

Sources d'organisation et, par voie de conséquence, facteurs de classement, de modélisation ou de synthèse, les choix graphiques d'un chercheur n'influencent pas seulement la présentation formelle des résultats ou leur réception par les lecteurs; ils sont susceptibles d'avoir une incidence sur l'analyse et la construction des données ou des objets de recherche ${ }^{4}$. Les quatre ethnologues de Minot en ont d'ailleurs parfaitement conscience puisque, comme le souligne Françoise Zonabend, elles abandonnent leur fichier « matières » au profit des récits de vie dès qu'elles renoncent au projet initial de monographie villageoise. Mais les choix scripturaux ne se réduisent pas à des stratégies intentionnelles : sur le long terme, les goûts graphiques de Marcel Griaule ont tellement pesé sur la mise en forme des « systèmes de pensée » étudiés qu'ils ont fini par façonner, de façon insidieuse, une partie des mythes, des taxinomies et des signes attribués aux Dogon. C'est ce que démontre Éric Jolly en examinant l'ensemble des textes de Griaule, à toutes les étapes de leur fabrication.

\section{Processus scripturaux et trajectoires individuelles}

7 Hérités en partie des études littéraires, les travaux antérieurs sur l'écriture ethnographique avaient tendance à privilégier, dans leur analyse, les procédés rhétoriques de persuasion employés à des fins d'autorité textuelle ${ }^{5}$. De telles recherches négligeaient, en revanche, l'évolution scripturale d'un chercheur ainsi que les différentes strates d'écriture qu'il a pu utiliser, en s'interdisant, de ce fait, toute analyse diachronique de la fabrication d'un texte. Aussi les articles de ce dossier suivent-ils plutôt les traces de travaux récents, en particulier ceux de Giordana Charuty qui, à partir de papiers d'archives foisonnants, reconstitue l'évolution conjointe de l'écriture et du parcours intellectuel d'Ernesto De Martino, éclairant ainsi les silences de son œuvre scientifique ${ }^{6}$. Ils s'inspirent également d'études originales sur les archives ethnographiques ${ }^{7}$, les notes de travail ${ }^{8}$, les biographies ${ }^{9}$ et les écrits ordinaires ${ }^{10}$. Les articles de ce dossier exploitent, en effet, tous les papiers disponibles afin d'analyser en parallèle les choix graphiques d'un chercheur, la genèse de ses textes, son itinéraire intellectuel et sa trajectoire professionnelle. 


\section{NOTES}

1. Cf., notamment, Le Métier d'anthropologue. Sens et liberté de Marc Augé (Paris, Galilée, 2006) et, en particulier, la troisième partie intitulée « L'écriture ».

2. Vincent Debaene a tenté de retracer ces attirances, tensions et oppositions entre anthropologie et littérature dans L'Adieu au voyage. L'ethnologie française entre science et littérature (Paris, Gallimard, 2010).

3. La démarche réflexive de Françoise Zonabend sur l'un de ses terrains français permet ainsi d'échapper à une analyse trop souvent limitée aux écrits produits dans un contexte colonial et/ou exotique. Dans l'Avant-propos d'un dossier français inédit consacré à l'écriture ethnographique, Jean Jamin et Françoise Zonabend soulignaient que les premières analyses critiques des textes ethnographiques coïncidaient avec la revisite des terrains lointains de leurs auteurs, dans un contexte postcolonial autorisant ou même favorisant de tels réexamens (cf. Jean Jamin \& Françoise Zonabend, "Avant-propos ", Études rurales, 1985, 97-98: Le texte ethnographique : 9-12, ici p. 9).

4. Sur les propriétés cognitives des productions graphiques, cf. : Jack Goody, La Raison graphique. La domestication de la pensée sauvage, Paris, Minuit, 1979; Jean-Marc Besse, « Nature, formes et productivité de la visualisation dans la construction des savoirs », in Christian Jacob, ed., Lieux de savoir, 2 : Les mains de l'intellect, Paris, Albin Michel, 2011 : 580-587 ; Rafael Mandressi, « Gestes et formes de l'écriture savante », in Christian Jacob, ed., ibid. : 283-289.

5. Cf. : James Clifford, «De l'autorité en ethnographie ", L'ethnographie, 1983, 90-91: 87-118 ; James Clifford \& George E. Marcus, eds, Writing Culture. The Poetics and Politics of Ethnography, Berkeley, University of California Press, 1986 ; Clifford Geertz, Ici et là-bas. L'anthropologue comme auteur, Paris, Métailié, 1996 [1988 pour l'édition américaine].

6. Giordana Charuty, Ernesto De Martino. Les vies antérieures d'un anthropologue, Marseille, Parenthèses-MMSH, 2009.

7. Dossier «Archives et anthropologie », établi et présenté par Jean Jamin \& Françoise Zonabend, Gradhiva, 2001-2002, 30-31 ; Antoinette Molinié \& Marie-Dominique Mouton, "L'ethnologue aux prises avec les archives - Introduction », Ateliers d'anthropologie, 2008, 32 [http://ateliers.revues.org/1093].

8. Roger Sanjek, ed., Fieldnotes. The Makings of Anthropology, Ithaca, Cornell University Press, 1990 ; Langage \& Société, 2009, 127 : Écritures scientifiques. Carnets, notes, ébauches. Parmi les travaux de génétique textuelle, on peut citer également le numéro de Langue française, 2007, 155: Avant le texte: les traces de l'élaboration textuelle, dirigé par Irène Fenoglio \& Lucile Chanquoy.

9. Daniel Fabre, Jean Jamin \& Marcello Massenzio, «Jeu et enjeu ethnographique de la bio- graphie », L'Homme, 2010, 195-196 : 7-20 ; Marc-Antoine Kaeser, « La science vécue : les potentialités de la biographie en histoire des sciences ", Revue d'histoire des sciences humaines, 2003, $8: 139-160$. 
10. Daniel Fabre, ed., Écritures ordinaires, Paris, Centre Georges-Pompidou-PoL, 1993 ; Aïssatou Mbodj-Pouye, "Tenir un cahier dans la région cotonnière du Mali : support d'écriture et rapport à soi ", Annales HSS, 2009, 4 : 855-885.

\section{AUTEURS}

ÉRIC JOLLY

Centre national de la recherche scientifique

\section{MARIANNE LEMAIRE}

Centre d'études des mondes africains (CEMAf), Ivry-sur-Seine 